\title{
ANÁLISIS DEL DOLOR MUSCULOESQUELÉTICO EN TRABAJADORES SANITARIOS DURANTE UNA JORNADA LABORAL
}

\author{
ANALYSIS OF MUSCULOSKELETAL PAIN IN HEALTHCARE WORKERS DURING A \\ WORKDAY
}

\author{
Jonathan Osorio-Vasco* \\ Yordán Rodríguez ${ }^{* *}$
}

\begin{abstract}
Resumen: En este artículo se analiza el comportamiento del dolor musculoesquelético en 21 trabajadores de un hospital de Medellín, Colombia. Mediante un cuestionario los trabajadores reportaron la percepción de dolor musculoesquelético por partes del cuerpo durante la primera y la última hora de una jornada laboral de 8 horas. Se analizó la intensidad y la frecuencia de dolor musculoesquelético y se calculó el porcentaje de cambio de dolor entre el inicio y el final de la jornada laboral para cada segmento corporal, y se clasificó la intensidad y el porcentaje de cambio de dolor en tres niveles (leve, moderado y severo). Se obtuvo que las partes del cuerpo con mayor intensidad de dolor fueron espalda baja, muslo-cadera, pierna, hombro y rodilla del lado derecho del cuerpo. Mientras que las partes del cuerpo con mayor frecuencia de dolor al inicio fueron brazo izquierdo (15) y al final pierna (15) y tobillo-pie (15) izquierdos. Por otro lado, los segmentos corporales que tuvieron un aumento significativo entre el inicio y el final de la jornada laboral fueron hombro izquierdo ( $\mathrm{Z}=-$ 2,100, $\mathrm{p}=0,036)$, muslo-cadera izquierda $(\mathrm{Z}=-2,273, \mathrm{p}=0,023)$ y tobillo-pie derecho $(\mathrm{Z}=-2,395$, $\mathrm{p}=0,017)$. El nivel de dolor fue de moderado a severo en el $66 \%$ de las partes del cuerpo y el porcentaje de aumento de dolor fue entre moderado y severo en el $65 \%$ de las partes del cuerpo. Este estudio muestra cómo las actividades laborales ejecutadas por una población de trabajadores sanitarios pueden provocar un aumento del dolor musculoesquelético en determinadas zonas corporales.
\end{abstract}

Palabras clave: Dolor musculoesquelético, dolor de la región lumbar, salud ocupacional, riesgos laborales, enfermedades laborales, sector de atención de salud.

\begin{abstract}
This article analyzes the behavior of musculoskeletal pain in 21 workers of a hospital in Medellín, Colombia. Using a questionnaire, the workers reported musculoskeletal pain perception by body part during the first and last hour of an 8-hour workday. The intensity and frequency of musculoskeletal pain were analysed. Also the percentage change of pain between the beginning and the end of the workday was calculated for each body segment. The intensity and percentage change of pain were classified into three levels (mild, moderate and severe). It was obtained that the body parts with the highest pain intensity were low back, thigh-hip, leg, shoulder and knee on the right side of the body. In contrast, the body parts with the highest frequency of pain at the beginning were the left arm (15) and at the end the left leg (15) and ankle-foot (15). On the other hand, the body segments that had a significant increase between the beginning and the end of the working day were left shoulder $(\mathrm{Z}=-$
\end{abstract}

*Universidad de Antioquia. Medellín, Colombia. Correo electrónico: jonathan.osorio@udea.edu.co. Orcid: https://orcid.org/oooo-0002-3062-5736. Autor de correspondencia.

**Universidad de Antioquia. Medellín, Colombia. Correo electrónico: yordan.rodriguez@udea.edu.co. Orcid: https://orcid.org/o0oo-0002-0079-4336 
2.100, $\mathrm{p}=0.036)$, left thigh-hip $(\mathrm{Z}=-2.273, \mathrm{p}=0.023)$ and right ankle-foot $(\mathrm{Z}=-2.395, \mathrm{p}=0.017)$. The pain level was moderate to severe in $66 \%$ of the body parts, and the percentage of increased pain was between moderate and severe in $65 \%$ of the body parts. This study shows how work activities performed by a population of healthcare workers can lead to increased musculoskeletal pain in specific body areas.

Keywords: Musculoskeletal pain, low back pain, occupational health, occupational risks, occupational diseases, health care sector.

Recepción: 20.05.2021 / Revisión: 02.06.2021 / Aceptación: 19.07.2021

\section{Introducción}

Los desórdenes musculoesqueléticos (DME) afectan a nivel mundial a 1700 millones de personas (Organización Mundial de la Salud, [OMS], 2021, 8 de febrero) y están relacionados con la alteración de la estructura corporal como: los músculos, articulaciones, tendones, ligamentos, nervios, huesos y sistema circulatorio causados por el entorno o ambiente donde se trabaja (Agencia Europea para la Seguridad y la Salud en el Trabajo, 2007). En los países desarrollados, las enfermedades musculoesqueléticas son una de las causas principales de ausentismo laboral, donde solo son superadas por enfermedades de corta duración como las respiratorias (Woolf \& Pfleger, 2003).

Estas enfermedades usualmente están precedidas por síntomas de dolor, el cual es frecuente en la aparición de enfermedades musculoesqueléticas (Trouvin \& Perrot, 2019). El dolor es definido por la Asociación Internacional para el Estudio del Dolor (International Association for the Study of Pain, IASP, por sus siglas en inglés) como: "Una experiencia sensorial y emocional desagradable asociada a un daño tisular real o potencial, o similar a este.”(International Association for the Study of Pain [IASP], 2017).

Según la cuarta encuesta de condiciones de trabajo realizada en Europa, el 25\% de los trabajadores se quejan de dolor en la espalda baja, el 23\% de trabajadores reporta dolor muscular y el $45 \%$ de trabajadores reporta trabajar en posiciones que causan dolor (ParentThirion et al., 2007). Por otro lado, en Centro América (Costa Rica, El Salvador, Honduras, Nicaragua y Panamá) se ha encontrado una alta prevalencia de dolor en trabajadores económicamente activos, principalmente en el segmento corporal cervical-dorsal por encima del segmento lumbar o las extremidades superiores (Rojas et al., 2015). En Colombia, según la II Encuesta Nacional de Condiciones de Seguridad y Salud en el Trabajo, el 25,46\% de los trabajadores encuestados reportó como factor de riesgo posiciones que pueden producir cansancio o dolor en algún segmento corporal y las lesiones musculoesqueléticas son el 90\% de la tendencia de enfermedades laborales hasta el año 2012 (Ministerio del Trabajo, 2013).

Los trabajadores del sector sanitario no están ajenos a la aparición de síntoma de dolor musculoesquelético relacionado con el trabajo. Por ejemplo, en un meta análisis realizado se muestra cómo las enfermeras y las auxiliares de enfermería reportaron síntoma de dolor en el cuello, las extremidades superiores, las extremidades inferiores y la espalda (Bernal et al., 2015). En otros estudios, se encontró que algunas actividades de trabajo desarrolladas por 
enfermeras y personal sanitario de los hospitales se relacionaron con dolor en el cuello, espalda baja, rodillas, entre otros (Meijsen \& Knibbe, 2007; Mirmohammadi et al., 2015; Omokhodion et al., 2000).

Comúnmente los estudios relacionados con el dolor reportado por el personal sanitario se realizan de manera retrospectiva (ej. la última semana, los últimos tres meses o los últimos 12 meses) mediante el uso de cuestionarios. Aunque estos estudios aportan información valiosa, no permiten cuantificar el efecto de las actividades laborales en los cambios de dolor musculoesquelético entre el inicio y final de una jornada laboral.

El objetivo de este estudio es analizar la percepción de dolor musculoesquelético en trabajadores sanitarios entre el inicio y final de una jornada laboral de 8 horas en un hospital de Medellín, Colombia, identificando los segmentos corporales con mayor frecuencia e intensidad de dolor musculoesquelético y cuantificando los cambios de dolor ocurridos entre el inicio y final de la jornada laboral.

\section{Materiales y métodos}

Estudio transversal donde se evaluó la percepción del dolor musculoesquelético en 21 trabajadores (12 mujeres, 9 hombres) de un hospital de Medellín Colombia, al inicio y al final de una jornada laboral de 8 horas. Estos trabajadores se desempeñaban en siete puestos de trabajo diferentes del hospital. El número de trabajadores por puesto se distribuyó de la siguiente manera: tres en distribución y dietética, tres en farmacia, cinco en Unidad de Cuidados Intensivos (UCI), tres en lavandería, tres en mantenimiento, tres en hospitalización y uno de limpieza.

La selección de los participantes se realizó tomando en cuenta la cantidad de trabajadores asignados a cada puesto de trabajo. En los puestos de trabajo donde trabajaban entre uno y tres trabajadores, fueron seleccionados todos los trabajadores. En los casos donde se desempeñaban más de tres trabajadores en el puesto, la selección fue aleatoria. Para la participación en el estudio, los trabajadores debían ejecutar sus actividades en alguno de los puestos seleccionados, estar presentes el día de la recolección de datos y manifestar su participación voluntaria en el estudio para lo cual firmaron un consentimiento informado.

Para evaluar el dolor musculoesqueléticos se les preguntó a los trabajadores que indicaran el nivel de dolor usando la escala visual análoga, representada en una línea 100 mm de longitud (Price et al., 1983), donde el o en el extremo izquierdo de la línea indicaba "no dolor" y el 100 al extremo derecho indicaba "máximo dolor". Esta escala ha sido empleada en diversos estudios, es de fácil aplicación, es sensible a los cambios en la percepción de dolor y los datos obtenidos con su uso pueden ser analizados con una gran variedad de técnicas estadísticas (Straker, 2005). Fueron evaluados 21 segmentos corporales usando como referencia el mapa del cuerpo de la norma ISO/TS 20646:2014 (International Organization for Standardization [ISO], 2014). Los segmentos corporales fueron: cuello, espalda alta, espalda baja y los segmentos derechos e izquierdos de hombros, codos, antebrazos, mano-muñecas, muslo-caderas, rodillas, piernas y tobillo-pies. 
El registro de la evaluación del dolor se realizó durante la primera hora de la jornada laboral. Se les explicó a los trabajadores cómo debían registrar el dolor percibido en ese momento del día, posteriormente se les pidió que marcaran con una línea vertical de color rojo sobre la escala visual análoga la percepción de dolor de cada segmento corporal. Este mismo procedimiento para el registro del dolor se ejecutó durante la última hora de trabajo.

Para el reporte estadístico de las variables sociodemográficas: sexo, edad, talla y peso se usaron estadísticos descriptivos tales como: promedio y desviación estándar. Para las variables de la percepción del dolor musculoesquelético al inicio y final de la jornada laboral por cada segmento corporal se usó: promedio, se calculó de la diferencia y la prueba de rangos con signo de Wilcoxon para hallar diferencias significativas. Para el cálculo del porcentaje promedio de cambio se usó la fórmula:

$$
\Delta \mathrm{D} \%=((\mathrm{D} 2-\mathrm{D} 1) / \mathrm{D} 1) \times 100 .
$$

Donde: D1 es el dolor musculoesquelético al inicio de la jornada laboral y D2 el dolor musculoesquelético al final de la jornada laboral.

Para mostrar los niveles de intensidad de dolor por segmento corporal de los 21 trabajadores, se establecieron tres rangos basados en el dolor musculoesquelético promedio registrado al final de la jornada laboral. Estos fueron los rangos establecidos (figura 2): dolor leve $\leq 10 \mathrm{~mm}$ (color verde); dolor moderado $>10 \mathrm{~mm} \mathrm{y} \leq 20 \mathrm{~mm}$ (color amarillo); y dolor severo $>20 \mathrm{~mm}$ (color rojo). Para la representación gráfica y generación de las figuras 1 y 2 del mapa del cuerpo, se empleó la plataforma de evaluación ergonómica desarrollada por Ergoyes (www.ergoyes.com).

Con base en el porcentaje promedio de cambio de dolor musculoesquelético, se establecieron tres rangos para evidenciar la magnitud en el cambio de dolor por segmento corporal. Estos fueron los rangos establecidos (figura 1): dolor leve $\leq 25 \%$, (color verde); dolor moderado $>25 \%$ y $\leq 50 \%$ (color amarillo); y dolor severo > 50\%, (color rojo).

También se analizó la frecuencia de dolor musculoesqueléticos por región corporal, al inicio y final de la jornada laboral. Para este análisis, se consideró presencia de dolor si el trabajador marcaba un valor mayor a cero en la escala visual análoga, mientras que si marcaba cero, se consideró que no tenía dolor.

\section{Resultados y discusión}

\section{Características sociodemográficas}

En total participaron en el estudio 21 trabajadores (12 mujeres y 9 hombres), con edad promedio de 35,19 años ( $\mathrm{DS}=9,91)$; estatura promedio de 165,4 cm (DS=9,91); peso promedio $71,82 \mathrm{~kg}$ (DS=12,64).

\section{Dolor musculoesquelético}

Intensidad y nivel de dolor musculoesquelético

En la tabla 1 se muestran los niveles de intensidad promedio al final de la jornada laboral. De 
las partes analizadas la espalda baja $(26,47)$, fue el de mayor intensidad, seguido de los segmentos corporales: muslo-cadera derecha $(23,85)$, pierna derecha $(22,04)$, hombro derecho $(20,28)$ y rodilla derecha $(20,19)$. Debe señalarse, que aunque la espalda baja fue el segmento corporal de mayor promedio de dolor, no se encontraron diferencias significativas entre el inicio y el final de la jornada laboral, siendo el porcentaje de cambio de dolor bajo $(3,22 \%)$.

Algunos estudios han identificado que el dolor musculoesquelético en la espalda baja es un segmento corporal con alta prevalencia entre el personal sanitario (Bejia et al., 2005; Bernal et al., 2015; Jellad et al., 2013; Mirmohammadi et al., 2015; Omokhodion et al., 2000), sin embargo, son estudios que se han realizado a través de cuestionarios donde se pregunta sobre la presencia de dolor durante un periodo de tiempo determinado (ej. últimos 12 o 3 meses).

El lado derecho del cuerpo, junto al cuello, la espalda alta y baja, fueron en la población estudiada los segmentos corporales de mayor nivel de dolor, puesto que a excepción del antebrazo, los otros segmentos corporales analizados presentaron un nivel de dolor musculoesquelético entre moderado y severo al final de la jornada laboral, destacando el hombro, el muslo-cadera, la rodilla y la pierna (figura 1).

Figura 1. Nivel promedio de dolor musculoesquelético registrado al final de la jornada laboral $(n=21)$.

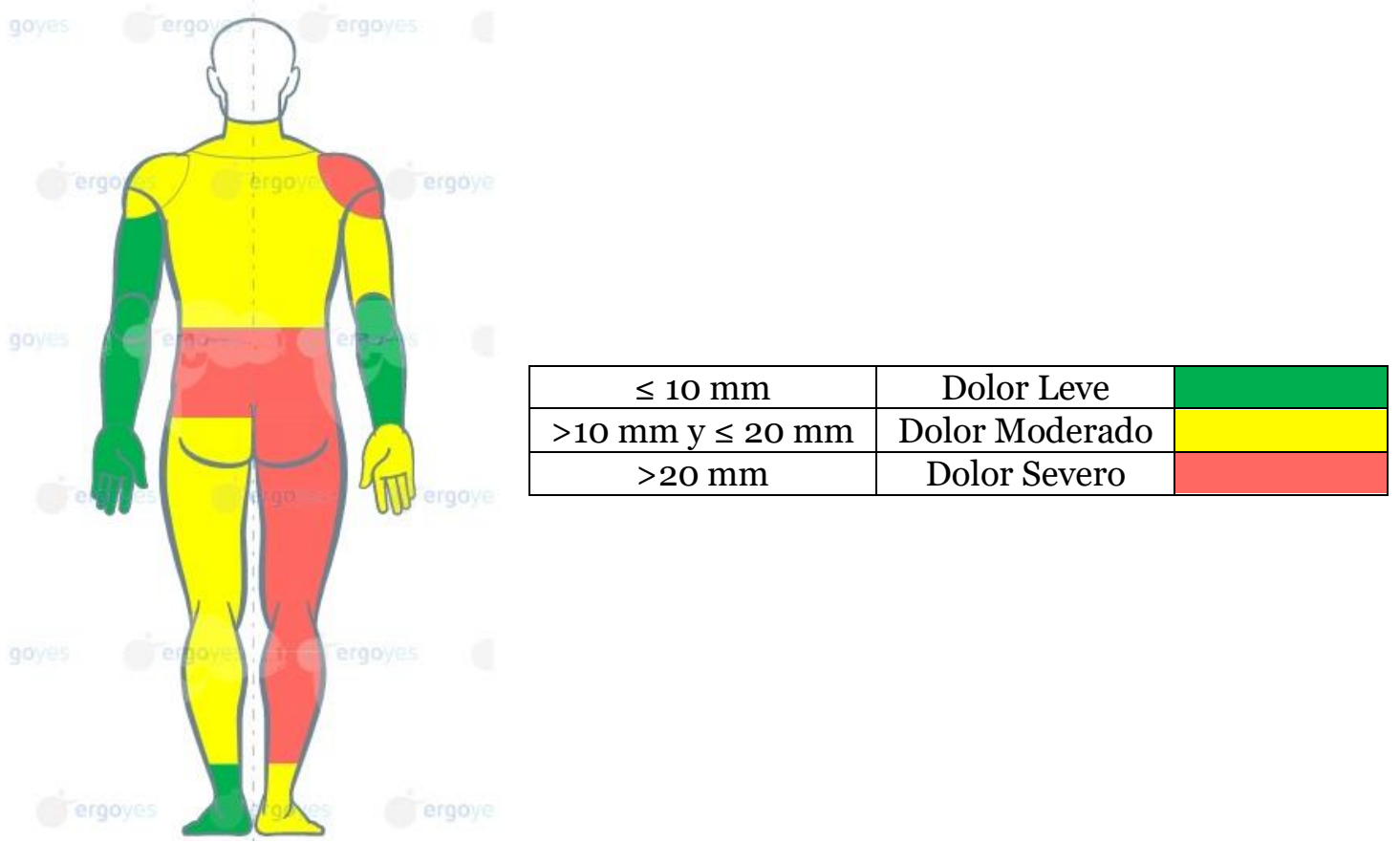

Fuente. Elaborado con la plataforma de evaluación ergonómica Ergoyes (www.ergoyes.com). 


\section{Porcentaje promedio de cambio de dolor musculoesquelético}

Los segmentos corporales con mayor aumento de porcentaje promedio de dolor musculoesquelético durante la jornada laboral fueron: hombro izquierdo (541,67\%), brazo izquierdo (352\%), tobillo-pie derecho (311,36\%), tobillo-pie izquierdo (251,85\%) y brazo derecho (131,64\%) (tabla 1). A pesar de que en 19 segmentos corporales aumentó porcentualmente el dolor musculoesquelético entre el inicio y final de la jornada laboral, donde se incluye el segmento corporal de la espalda baja (tabla 1), son los segmentos corporales de las extremidades superiores e inferiores con mayor porcentaje de cambio promedio durante la jornada laboral. Este resultado llama la atención porque en el personal sanitario es común que el segmento corporal de la espalda baja es uno de los segmentos corporales más reportados (Jellad et al., 2013; Mirmohammadi et al., 2015; Omokhodion et al., 2000). Estos resultados también concuerdan con que el segmento corporal de los hombros en personal sanitario es uno de los más prevalentes (Bernal et al., 2015; Mirmohammadi et al., 2015). Con base al porcentaje de cambio, 10 segmentos corporales tuvieron cambios severos de dolor, especialmente en las extremidades superiores e inferiores del cuerpo, mientras que 2 segmentos corporales que fueron las manos tuvieron cambios moderados de dolor y finalmente 7 segmentos corporales donde se encuentra la espalda alta y baja tuvieron cambios moderados de dolor (figura 2).

Figura 2. Porcentaje de cambio promedio de dolor musculoesquelético $(n=21)$.

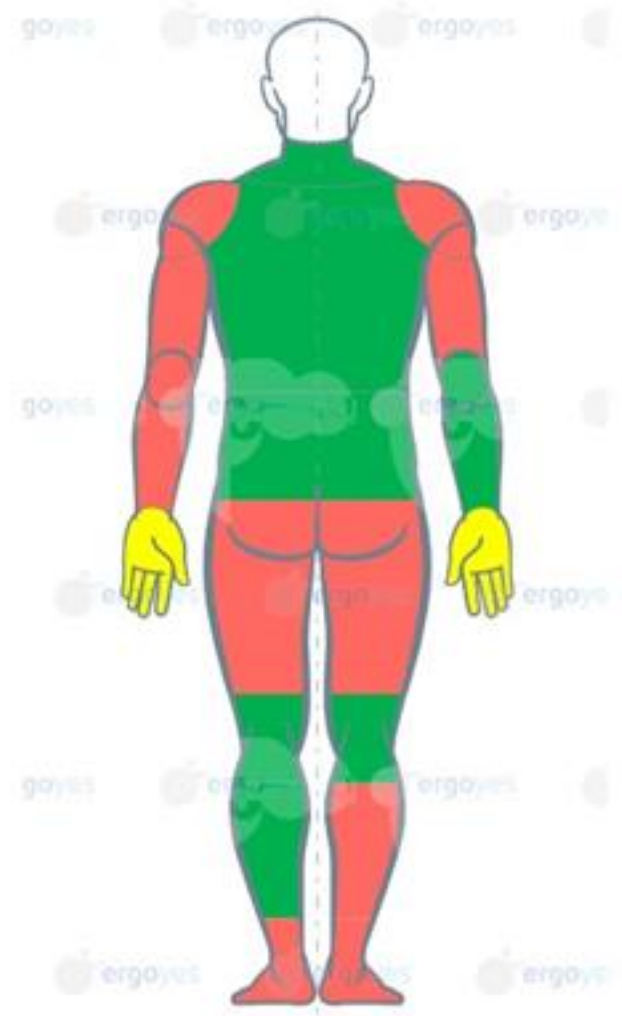

\begin{tabular}{|c|c|c|}
\hline$\leq 25 \%$ & Dolor Leve & \\
\hline$>25 \% \mathrm{y} \leq 50 \%$ & Dolor Moderado & \\
\hline$>50 \%$ & Dolor Severo & \\
\hline
\end{tabular}

Fuente. Elaborado con la plataforma de evaluación ergonómica Ergoyes (www.ergoyes.com). 
Cambio de dolor musculoesquelético entre el inicio y final de la jornada de laboral

El síntoma de dolor musculoesquelético aumentó en 19 de 21 segmentos corporales evaluados entre el inicio y final de la jornada laboral en trabajadores del sector sanitario de un hospital (tabla 1). Sin embargo, el dolor musculoesquelético solo aumentó de manera significativa en los segmentos corporales: hombro izquierdo $(Z=-2,100, p=0,036)$, muslocadera izquierda $(Z=-2,273, p=0,023)$ y tobillo-pie derecho $(Z=-2,395, p=0,017)$. Solo los segmentos corporales del codo y antebrazo derecho disminuyeron la intensidad del dolor.

Tabla 1. Cambio promedio de dolor musculoesquelético y porcentaje de aumento del dolor entre el inicio y final de la jornada. $\mathrm{P}<0,05(\mathrm{n}=21)$.

\begin{tabular}{|c|c|c|c|c|c|c|c|}
\hline \multirow{2}{*}{$\begin{array}{l}\text { Segmento } \\
\text { corporal }\end{array}$} & \multirow{2}{*}{$\begin{array}{l}\text { Lado del } \\
\text { cuerpo }\end{array}$} & \multirow{2}{*}{$\begin{array}{c}\text { Dolor } \bar{X} \\
\text { inicial }\end{array}$} & \multirow{2}{*}{$\begin{array}{l}\text { Dolor } \bar{X} \\
\quad \text { final }\end{array}$} & \multirow{2}{*}{$\Delta \bar{X}$} & \multirow{2}{*}{$\Delta \boldsymbol{D} \bar{X} \%$} & \multicolumn{2}{|c|}{$\begin{array}{c}\text { Prueba } \\
\text { Wilcoxon }\end{array}$} \\
\hline & & & & & & $\mathbf{Z}$ & $\mathbf{P}$ \\
\hline Cuello & - & 12,28 & 13,80 & 1,52 & 12,38 & $-0,534$ & 0,594 \\
\hline Espalda alta & - & 16,47 & 17,00 & 0,53 & 3,22 & $-0,722$ & 0,470 \\
\hline Espalda baja & - & 21,9 & 26,47 & 4,57 & 20,87 & $-0,513$ & 0,608 \\
\hline \multirow{2}{*}{ Hombro } & Derecho & 10,28 & 20,28 & 10,00 & 97,28 & $-1,274$ & 0,203 \\
\hline & Izquierdo & 2,04 & 13,09 & 11,05 & 541,67 & $-2,100$ & 0,036 \\
\hline \multirow{2}{*}{ Brazo } & Derecho & 4,52 & 10,47 & 5,95 & 131,64 & $-0,841$ & 0,400 \\
\hline & Izquierdo & 1,00 & 4,52 & 3,52 & 352,00 & $-1,153$ & 0,249 \\
\hline \multirow{2}{*}{ Codo } & Derecho & 4,33 & 3,85 & $-0,48$ & $-11,09$ & $-0,315$ & 0,752 \\
\hline & Izquierdo & 0,71 & 2,09 & 1,38 & 194,37 & $-0,730$ & 0,465 \\
\hline \multirow{2}{*}{ Antebrazo } & Derecho & 9,47 & 6,61 & $-2,86$ & $-30,20$ & $-0,141$ & 0,888 \\
\hline & Izquierdo & 3,57 & 6,47 & 2,90 & 81,23 & $-1,820$ & 0,069 \\
\hline \multirow{2}{*}{$\begin{array}{l}\text { Mano } \\
\text { muñeca }\end{array}$} & Derecha & 11,80 & 16,28 & 4,48 & 37,97 & $-0,070$ & 0,944 \\
\hline & Izquierda & 6,61 & 9,47 & 2,86 & 43,27 & $-1,534$ & 0,125 \\
\hline \multirow{2}{*}{$\begin{array}{l}\text { Muslo } \\
\text { cadera }\end{array}$} & Derecha & 11,8 & 23,85 & 12,05 & 102,12 & $-1,887$ & 0,059 \\
\hline & Izquierda & 11,85 & 18,04 & 6,19 & 52,24 & $-2,273$ & $\mathbf{0 , 0 2 3}$ \\
\hline \multirow{2}{*}{ Rodilla } & Derecha & 18,38 & 20,19 & 1,81 & 9,85 & $-0,489$ & 0,625 \\
\hline & Izquierda & 12,85 & 13,23 & 0,38 & 2,96 & $-0,551$ & 0,582 \\
\hline \multirow{2}{*}{$\begin{array}{l}\text { Pierna } \\
\text { derecha }\end{array}$} & Derecha & 13,47 & 22,04 & 8,57 & 63,62 & $-1,373$ & 0,170 \\
\hline & Izquierda & 12,66 & 13,85 & 1,19 & 9,4 & $-0,523$ & 0,533 \\
\hline \multirow{2}{*}{ Tobillo pie } & Derecho & 3,61 & 14,85 & 11,24 & 311,36 & $-2,395$ & $\mathbf{0 , 0 1 7}$ \\
\hline & Izquierdo & 2,7 & 9,5 & 6,8 & 251,85 & $-1,122$ & 0,262 \\
\hline
\end{tabular}

Fuente. Elaboración propia.

Este estudio evaluó el dolor musculoesquelético en trabajadores sanitarios durante una jornada laboral entre el inicio y final, a diferencia de otros estudios que evaluaron los segmentos corporales del personal sanitario a través de cuestionarios que analizaban preguntas de prevalencia de dolor musculoesquelético durante los últimos 12 meses (Jellad et al., 2013; Mirmohammadi et al., 2015; Omokhodion et al., 2000) o 3 meses (Meijsen \& Knibbe, 2007). Este estudio permitió evidenciar en qué magnitud aumenta el dolor 
musculoesquelético del personal sanitario durante un día de trabajo en diferentes partes del cuerpo.

Los resultados de este estudio son importantes para dimensionar el comportamiento del dolor musculoesquelético en los diferentes segmentos coporales del personal sanitario que trabaja en hospitales, y así empezar a enfocar las acciones preventivas hacia las actividades que posiblemente aumentan el nivel y el cambio porcentual del dolor musculoesquelético.

Otro aspecto importante del estudio, es que logró realizar la evaluación del dolor en zonas que son críticas dentro de un hospital (UCI, hospitalización, distribución y dietética), donde los trabajadores que están en estas áreas constantemente se encuentran con niveles altos de ocupación. Por lo tanto, lograr la evaluación del dolor musculoesquelético entre el inicio y final de la jornada laboral cobra un alto valor al lograr tener la "fotografía" del comportamiento del dolor musculoesqeulético durante una jornada típica de trabajo.

\section{Frecuencia de dolor musculoesquelético}

En 8 partes del cuerpo la frecuencia de dolor musculoesquelético fue mayor al final de la jornada laboral, mientras que en 11 partes del cuerpo la frecuencia de dolor musculoesquelético fue mayor al inicio de la jornada laboral; solo en 2 partes del cuerpo la frecuencia fue igual al inicio y final.

En la figura 3 se observa que el brazo izquierdo (15) tuvo la mayor frecuencia al inicio de la jornada laboral, seguido del antebrazo izquierdo (14) y antebrazo derecho (13); mientras que el cuello (13), la espalda alta (13) y el brazo izquierdo (13) son los segmentos corporales con mayor frecuencia al final de la jornada laboral. Adicionalmente, el hombro derecho presentó la menor frecuencia de dolor musculoesquelético al inicio (7) y final (6) de la jornada laboral.

Figura 3. Frecuencia de dolor musculoesquelético al inicio y final de la jornada laboral en la parte superior del cuerpo $(n=21)$.

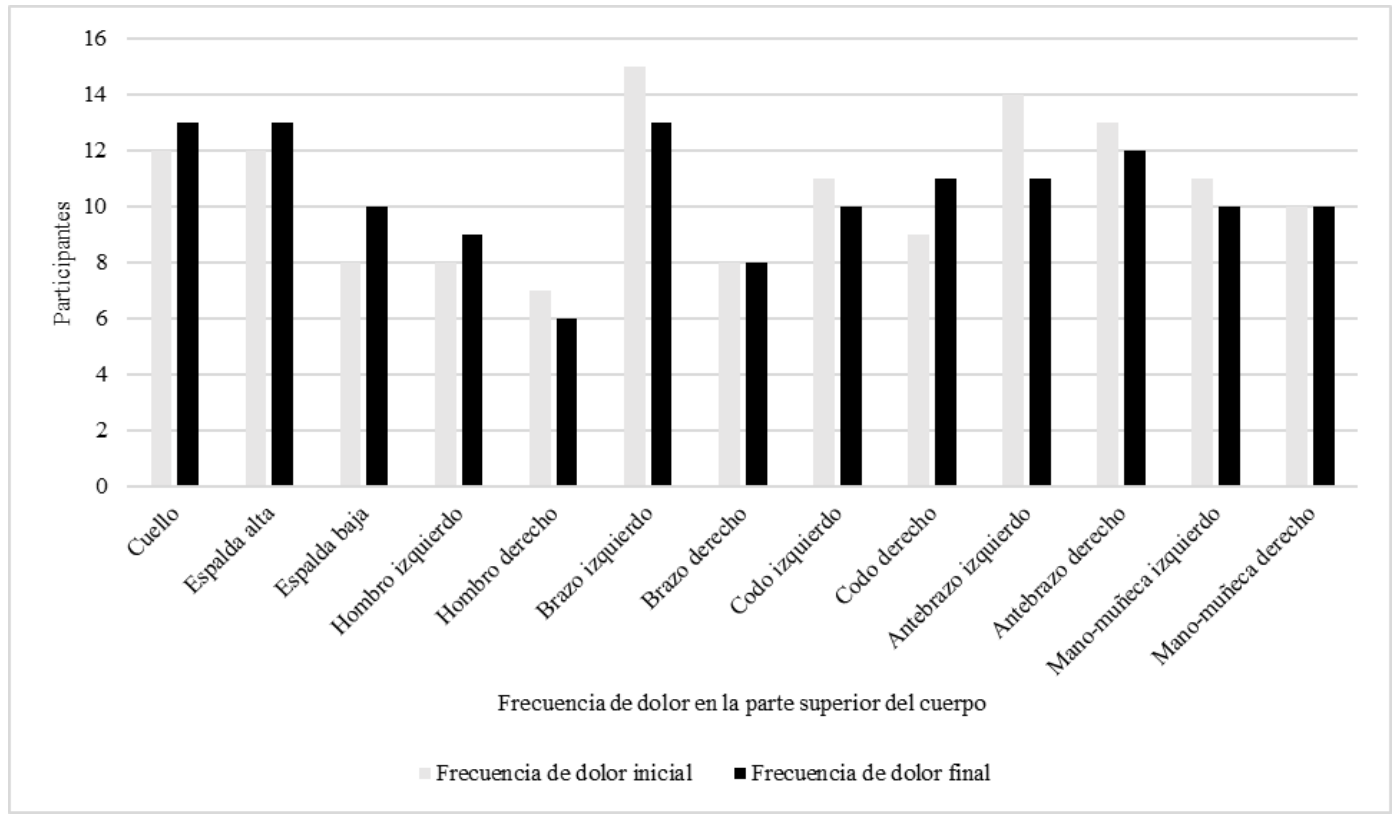


En la figura 4 se observa que la pierna derecha (13) fue la que tuvo mayor frecuencia al inicio de la jornada laboral, seguido de la rodilla derecha (12), la pierna izquierda (12) y el tobillo-pie izquierdo (12). La pierna izquierda (13) y el tobillo-pie izquierdo (13) tienen la mayor frecuencia de dolor muculoesquelético en la parte inferior del cuerpo al final de la jornada laboral, seguido de la pierna derecha (12) y tobillo-pie derecho (12).

Figura 4. Frecuencia de dolor musculoesquelético al inicio y final de la jornada laboral en la parte inferior del cuerpo $(n=21)$.

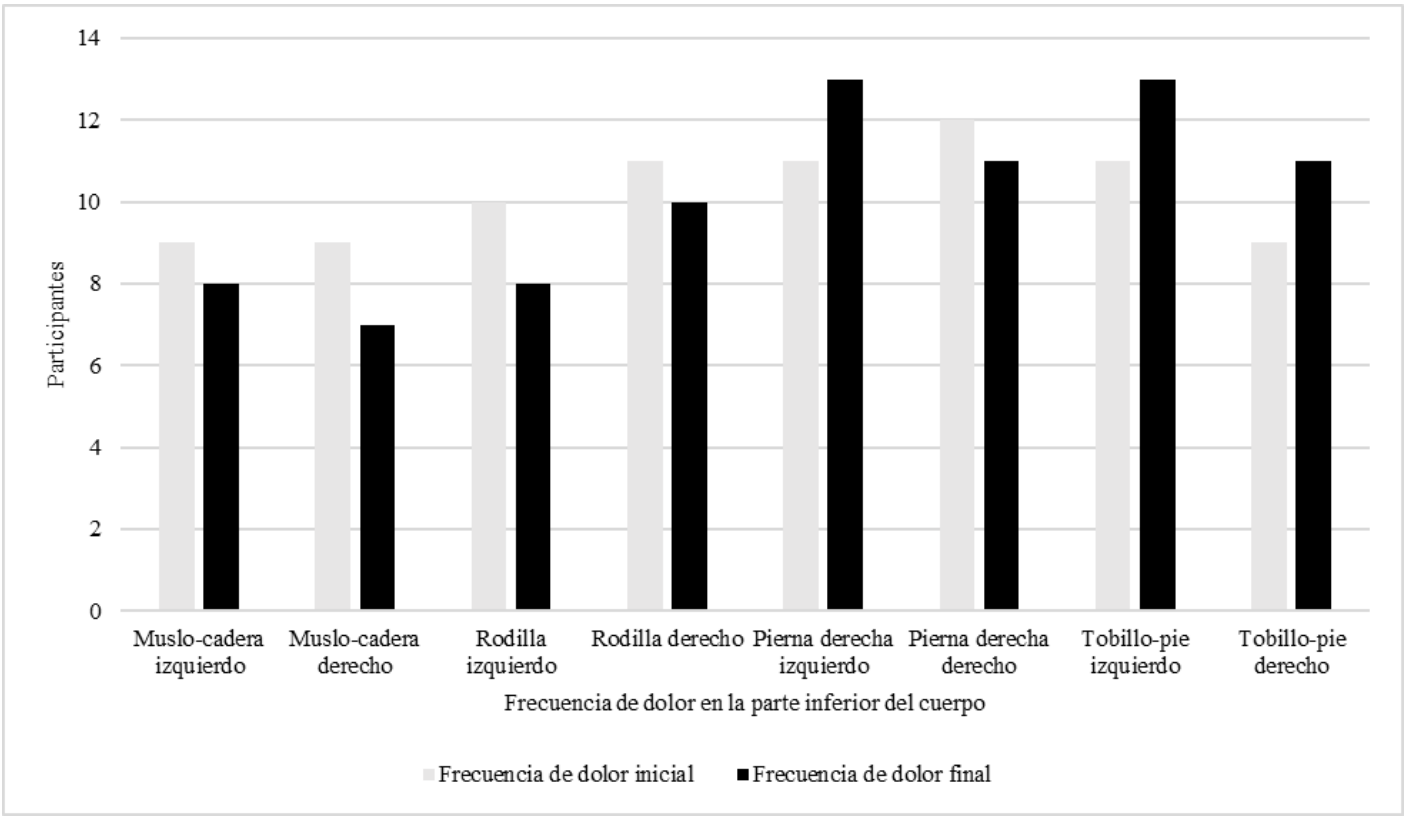

En este estudio se analizó la frecuencia de dolor musculoesqulético durante una jornada laboral en una muestra reducida de trabajadores sanitarios $(n=21)$. No obstante, los resultados son similares a a otros reportados con mayor tamaño de muestra en población de trabajadores sanitarios (Mirmohammadi et al., 2015; Ribeiro et al., 2017).

El análisis detallado por frecuencias, permite identificar cuántos trabajadores sanitarios presentan síntomas de dolor musculoesquelético en cada uno de los segmetnos corporales. Si bien el dolor musculoesquelético promedio aumentó en 20 segmentos corporales, no todos los trabajadores sanitarios presentaron aumento de dolor musculoesquelético al inicio y al final de la jornada laboral. Este resultado de la frecuencia de dolor musculoesquelético ayudará a identificar cuáles trabajadores o puestos de trabajo tienen cambio de dolor durante la jornada laboral y así direccionar actividades que ayuden a prevenir o disminuir el síntoma de dolor musculoesquelético.

Por último, este estudio evaluó el dolor musculoesquelético mediante la escala visual análoga, la cual presenta una elevada sensibilidad (Straker, 2005). Esta sensibilidad permitió detectar las variaciones de dolor en los diferentes segmentos corporales analizados. Se recomienda, que en próximos estudios se emplee la escala visual análoga para analizar el dolor en personal sanitario durante varios días y se estudie detalladamente la relación con las actividades que realizan; esto permitirá identificar cómo influyen cada una de las actividades laborales y posturas en el cambio de dolor musculoesquelético durante la jornada laboral. 
También, se considera que deberían incluirse otras variables extralaborales como: el tiempo de descanso, el tipo de transporte utilizado, actividad física, entre otras, que permitan comprender de una manera integral el comportamiento y factores asociados al dolor musculoesquelético.

\section{Conclusiones}

Este estudio muestra cómo las actividades laborales ejecutadas por una población de trabajadores sanitarios pueden provocar un aumento del dolor musculoesquelético en determinadas zonas corporales. Estos hallazgos, coinciden con otros estudios publicados e indican la necesidad de seguir investigando sobre los factores de riesgo en las actividades del sector sanitario y su relación con el dolor musculoesquelético para poder tomar acciones preventivas. 


\section{Referencias}

Agencia Europea para la Seguridad y la Salud en el Trabajo. (2007). Factsheet 72: Trastornos musculoesqueléticos de origen laboral en el cuello y en las extremidades superiores. https://osha.europa.eu/es/publications/factsheet-72-work-related-neck-and-upper-limbdisorders/view

Bejia, I., Younes, M., Jamila, H. B., Khalfallah, T., Salem, K. Ben, Touzi, M., Akrout, M., \& Bergaoui, N. (2005). Prevalence and factors associated to low back pain among hospital staff. Joint Bone Spine, 72(3), 254-259. https://doi.org/10.1016/j.jbspin.2004.06.001

Bernal, D., Campos-Serna, J., Tobias, A., Vargas-Prada, S., Benavides, F. G., \& Serra, C. (2015). Work-related psychosocial risk factors and musculoskeletal disorders in hospital nurses and nursing aides: A systematic review and meta-analysis. International Journal of Nursing Studies, 52(2), 635-648. https://doi.org/10.1016/j.ijnurstu.2014.11.003

International Association for the Study of Pain. (2017). IASP Terminology - Pain. December 14. https://www.iasp-pain.org/terminology?navItemNumber=576\#Pain

International Organization for Standardization. (2014). Technical specification ISO / TS 20646:2014 Ergonomics guidelines for the optimization of musculoskeletal workload (Vol. 2014). https://www.iso.org/standard/63231.html

Jellad, A., Lajili, H., Boudokhane, S., Migaou, H., Maatallah, S., \& Frih, Z. B. S. (2013). Musculoskeletal disorders among Tunisian hospital staff: Prevalence and risk factors. Egyptian Rheumatologist, 35(2), 59-63. https://doi.org/10.1016/j.ejr.2013.01.002

Meijsen, P., \& Knibbe, H. J. J. (2007). Work-related musculoskeletal disorders of perioperative personnel in the netherlands. AORN Journal, 86(2), 16. https://doi.org/10.1016/j.aorn.2007.07.011

Ministerio del Trabajo. (2013). Informe ejecutivo II encuesta nacional de condiciones de seguridad $y$ salud en el trabajo en el sistema general de riesgos. https://fasecolda.com/cms/wp-content/uploads/2019/o8/ii-encuesta-nacional-seguridadsalud-trabajo-2013.pdf

Mirmohammadi, S., Yazdani, J., Etemadinejad, S., \& Asgarinejad, H. (2015). A cross-sectional study on work-related musculoskeletal disorders and associated risk factors among hospital health cares. Procedia Manufacturing, 3(Ahfe), 4528-4534. https://doi.org/10.1016/j.promfg.2015.07.468

Omokhodion, F. O., Umar, U. S., \& Ogunnowo, B. E. (2000). Prevalence of low back pain among staff in a rural hospital in Nigeria. Occupational Medicine, 50(2), 107-110. https://doi.org/10.1093/occmed/50.2.107

Organización Mundial de la Salud. (2021, 8 de febrero). Trastornos musculoesqueléticos. https://www.who.int/es/news-room/fact-sheets/detail/musculoskeletal-conditions

Parent-Thirion, A., Macías, E. F., Hurley, J., \& Vermeylen, G. (2007). Fourth european working conditions

survey.

https://www.eurofound.europa.eu/es/publications/report/2007/workingconditions/fourth-european-working-conditions-survey

Price, D. D., McGrath, P. A., Rafii, A., \& Buckingham, B. (1983). The validation of visual analogue scales as ratio scale measures for chronic and experimental pain. Pain, 17(1), 45-56. https://doi.org/10.1016/0304-3959(83)90126-4

Ribeiro, T., Serranheira, F., \& Loureiro, H. (2017). Work related musculoskeletal disorders in primary health care nurses. Applied Nursing Research, 33, 72-77. https://doi.org/10.1016/j.apnr.2016.09.003 
Rojas, M., Gimeno, D., Vargas-Prada, S., \& Benavides, F. G. (2015). Dolor musculoesquelético en trabajadores de América Central: Resultados de la I encuesta centro americana de condiciones de trabajo y salud. Revista Panamericana de Salud Pública, 38(2), 120-128. https://iris.paho.org/handle/10665.2/10046

Straker, L. M. (2005). Body discomfort: Assessment tools. En W. Karwowski \& W. Marras (eds.), Occupational Ergonomics: Engineering and Administrative Controls (pp. 26-40). CRC Press.

Trouvin, A., \& Perrot, S. (2019). New concepts of pain. Best Practice \& Research Clinical Rheumatology, 33(3), 1-10. https://doi.org/10.1016/j.berh.2019.04.007

Woolf, A. D., \& Pfleger, B. (2003). Burden of major musculoskeletal conditions. Bulletin of the World Health Organization, 81(9), 646-656. https://doi.org/10.1590/So04296862003000900007 